\title{
The new approach of mathematical postprocessing of multidetector computed tomography results in pancreatic cancer diagnosis in patients with chronic pancreatitis
}

\author{
Konstantin LESKO ${ }^{* 1}$, Julia KULEZNEWA', Dmitry BORDIN² ${ }^{2}$, Elena DUBTSOVA ${ }^{2}$, Natalia ORLOVA', \\ Elena TYLYAEVA ${ }^{2}$, Marina MALYKH ${ }^{2}$, Boris NIKITIN', Lyudmila VINOKUROVA ${ }^{2}$ \\ 'Radiology Department, The Loginov Moscow Clinical Scientific Center, Moscow, Russia \\ ${ }^{2}$ Pancreatic and Biliary Tract Diseases Department, The Loginov Moscow Clinical Scientific Center, Moscow, Russia
}

Introduction: Our aim is to explore the diagnostic value of the new approach of pancreatic cancer (PC) diagnosis in patients with chronic pancreatitis (CP) by mathematical postprocessing of multidetector 128-row computed tomography (MDCT) results.

Methods: We analyzed results of contrast-enhanced MDCT from 122 patients with CP: 71 (58.2\%) males and 51 (41.8\%) females, mean age $53.7 \pm 13.03$ (30-83), who underwent MDCT from January 2018 to October 2019. All cases were pathologically proved by core-biopsy or surgery. We calculated median of enhancement gradient in Hounsfield units (HU) between region of interest and intact parenchyma (Mgrad) based on multiphase MDCT results. Correlation assessed by Pearson's correlation coefficient (r). The ROC curves were performed to evaluate diagnosis potential of Mgrad.

Results: There were 46 (37.7\%) patients with PC and 76 (62.3\%) patients with only CP. Mean Mgrad value was higher in PC-group $26.5 \pm 8.6 \mathrm{HU}$ than in CP-group - $13.9 \pm 5.1 \mathrm{HU}(\mathrm{p}=0.0001)$; Mgrad cut-off value for PC was $20 \mathrm{HU}(p=0.001)$. There was positive correlation of Mgrad in patients with PC $(\mathrm{r}=0.68, p=0.0001)$ and negative correlation in patients with CP $(\mathrm{r}=-0.68, p=0.0001)$. Sensitivity, specificity, accuracy, positive and negative predictive value of PC diagnosis were $89.1 \%, 96.1 \%, 93.4 \%, 93.2 \%, 93.6 \%$ for Mgrad and 69.5\%, 92.1\%, 83.6\%, 84.2\%, 83.3\% for MDCT, respectively; area under ROC for Mgrad was higher (0.927) than for MDCT (0.863). Conclusions: Mgrad assessment based on MDCT results is more effective than only MDCT for PC diagnosis in patients with CP. Mgrad value positively correlates with PC existence, therefore it could be useful for PC diagnosis. 